\title{
A new working principle for ac electro-hydrodynamic on-chip micro-pumps
}

\author{
Marco Stubbe $^{1}$, Moritz Holtappels ${ }^{2}$ and Jan Gimsa ${ }^{1,3}$ \\ ${ }^{1}$ Chair of Biophysics, Faculty of Biology, University of Rostock, D-18057 Rostock, Germany \\ ${ }^{2}$ Department of Biogeochemistry, Max Planck Institute for Marine Microbiology, \\ Celsiusstrasse 1, D-28359 Bremen \\ E-mail: jan.gimsa@uni-rostock.de
}

Received 21 August 2007, in final form 19 September 2007

Published 19 October 2007

Online at stacks.iop.org/JPhysD/40/6850

\begin{abstract}
Our new type of on-chip micro-pump exploits the ac electro-kinetic forces acting in the volume of a fluid in the presence of a temperature gradient. No mechanically movable parts are used. The velocity of the pump flow observed depends on the frequency and strength of the driving ac field and on the temperature gradient across the pump channel. An integrated heating element allows the temperature gradient to be adjusted. Both ac field electrodes and heating element are platinum structures processed on a glass chip. The pump-channel walls and cover are made from polymer and thin-glass, respectively. In this paper, we present measurements of the fluid velocity as functions of the medium conductivity $\left(0.1-1.3 \mathrm{~S} \mathrm{~m}^{-1}\right)$ and field frequency $(300 \mathrm{kHz}-52 \mathrm{MHz})$, voltage across the field-electrode voltage $\left(0-35 V_{\mathrm{rms}}\right)$ and the heating element $(1.1-3.6 \mathrm{~V})$. Velocities of up to $120 \mu \mathrm{m} \mathrm{s}^{-1}$ were observed in the pump channel. The advantage of our new design is an evenly shaped cross-section of the pump channel, which reduces the risk of the channel becoming clogged by debris.

Ac-electro-osmosis is not a predominant effect in our structures. Pumping could only be observed when the heating current and ac-pump field were applied simultaneously. The effects observed were simulated with the COMSOL Multiphysics program.
\end{abstract}

(Some figures in this article are in colour only in the electronic version)

\section{Introduction}

Recently, various types of integrated micro-analytical devices ( $\mu$-TAS) and lab-on chip-systems have been developed (Reyes et al 2002). These systems comprise micro-fluidic components with diverse potential applications. Some examples of systems and processes that employ this technology include drug dispensing systems, blood-cell separation devices, drug screening and chemical micro-reaction systems, or protein and DNA-chips, as well as the general actuation of liquids and their mixtures. A major problem in many of these fluidic microsystems is the directed transport of small volumes of liquid

3 Author to whom any correspondence should be addressed. media or particles. Electro-hydrodynamic pumps are at the focus of current developments, since they avoid the use of mechanical parts, which leads to an extended lifetime. The pumps allready known are based on three different principles. Besides the electrostatic principle (Bourouina et al 1997), inhomogeneous ac fields can be used for liquid pumping (Green et al 2000, 2002, Gonzales et al 2000, Ramos et al 1998, 2003, Wong et al 2004, Ajdari 2000). After the development of macroscopic travelling wave field applications (Ehrlich and Melcher 1982, Melcher et al 1966, 1967, 1969) the travelling wave principle has been rediscovered in order to transport small particles or to pump media in micro-systems (Fuhr et al 1990, 1991, 1992, 1994, Gimsa et al 1997, Hagedorn et al 1992, Müller et al 1993, Wang et al 1994). 


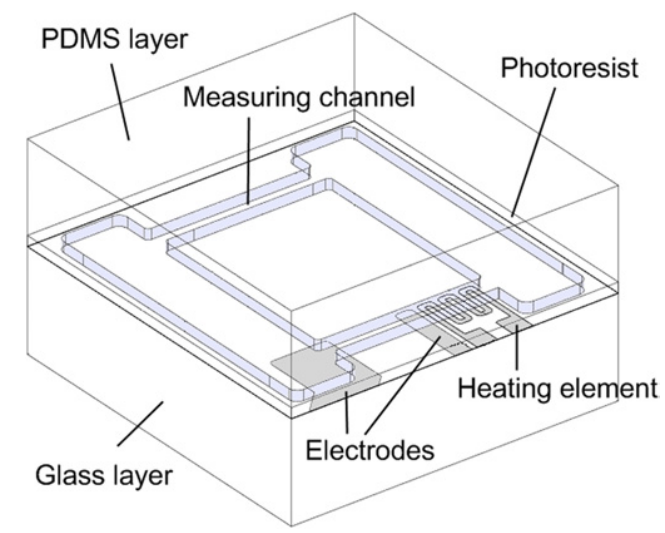

Figure 1. Schematic view of the pump. Electrodes and heating-meander were platinum structures on a glass-chip carrier. Channels were made from photoresist covered by a PDMS seal.

Micro pumps using inhomogeneous ac fields usually work with small inter-electrode gaps of around $25 \mu \mathrm{m}$ and up to $100 \mu \mathrm{m}$. In such small planar electrode systems, ac electroosmosis will be effective at frequencies below $1 \mathrm{MHz}$ as claimed by some authors (Green et al 2000, 2002, Gonzales et al 2000, Ramos et al 1998, 2003). Because of the small electrode size, high field strengths and strong field gradients may play a role.

We use larger geometries and higher field frequencies in our pumps. The original structures were originally designed for cell and particle dielectrophoresis (DP) experiments. In these structures, an inhomogeneous field was generated by a funnel-shaped dielectric body between two parallel electrodes. The distance between the electrodes was greater than $500 \mu \mathrm{m}$. Besides DP, pumping of the aqueous medium could also be observed (Gimsa and Holtappels, 2003). The pump effect was related to the temperature gradient generated by the resistive Joule-heating of the medium in the funnel-shaped channel. Joule-heating corresponds to the current density distribution determined by the channel geometry. We found that the pump effect was generated by the electric force acting inside the volume of the aqueous medium. This force is based on the gradient in electric medium properties caused by the induced temperature gradient.

In our new designs, we use a separate heating element to generate the temperature gradient independently of the ac-pump field and the pump-channel geometry. Accordingly, no special geometry of the pump channel is necessary, reducing the risk of channel clogging by particles or debris. In this paper, we present a microchip-system with a circular flow for easy and comparable velocity measurements. Two pump types with rectangular pump-channel profiles and a resistive heating element are presented. The ac field was either generated by two separate field electrodes or by a single electrode working against the potential of the heating element.

\section{Material and methods}

\subsection{Pump structures}

Figure 1 shows a three-dimensional view of the layered pump structure. A bottom thin-glass layer of $550 \mu \mathrm{m}$

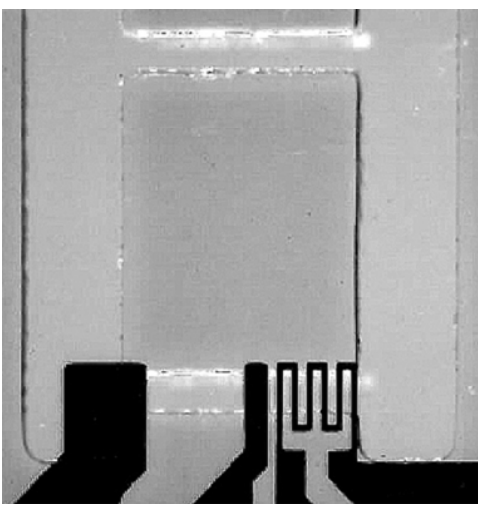

Figure 2. View of the indirectly heated pump structure with two field electrodes and a separate heating element. The field signal voltage was applied to the broader, left electrode whereas the narrower electrode was connected to ground. The meander structure is the heating element. Please note the homogeneous channel geometry.

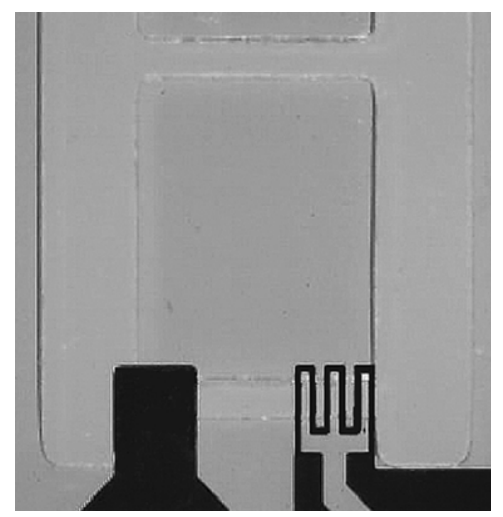

Figure 3. View of the directly heated pump structure with a single field electrode and a heating element also used as the counter ground electrode.

carries platinum electrodes and the heating element. The metallic structures were processed by photolithography on platinum plated (thickness about $100 \mathrm{~nm}$ ) thin-glass wafers (GeSIM GmbH, Grossermannsdorf, Germany). In a second step, a photoresist structure of approximately. $60 \mu \mathrm{m}$ in thickness was processed to form the channel-wall structures. For measurements, the channels were closed by a polydimethylsiloxane (PDMS) cover.

Figures 2 and 3 present an indirectly heated structure with two field electrodes and a separate resistive heating element and a directly heated structure with one field electrode using the heating element as the counter (ground) electrode.

Figure 4 gives details of the geometry of the heating element. The width of the meandering structure is about $30 \mu \mathrm{m}$. The heating element was driven by dc, with up to $3.6 \mathrm{~V}$. A current of about $6 \mathrm{~mA}$ was measured at $2.1 \mathrm{~V}$ and $2.1 \mathrm{~V}$ was used as a standard value. At $3.6 \mathrm{~V}$ temperatures up to $40^{\circ} \mathrm{C}$ were reached at a room temperature of $T_{0}=20^{\circ} \mathrm{C}$.

A thermal deterioration of the photoresist structures was observed at voltages above $4 \mathrm{~V}$. In pump structures with manufacturing errors hot spots could be observed at narrower sites of the heating structure. At these spots the aqueous pump solution could even start boiling. The reason for this effect was probably an auto feedback process induced by the low 


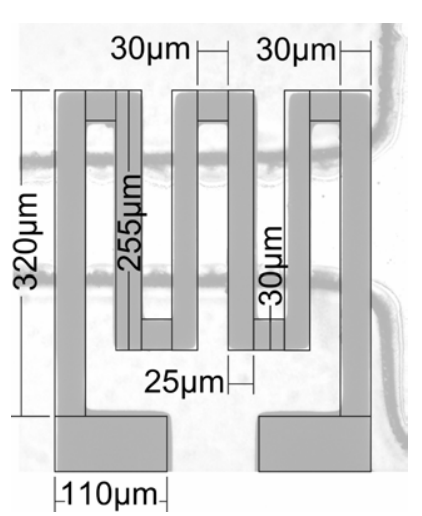

Figure 4. Dimensions of the heating element used in the pump chips. The metal-layer thickness is about $100 \mathrm{~nm}$. The sketch is a vector graphic on top of a microscopic image.

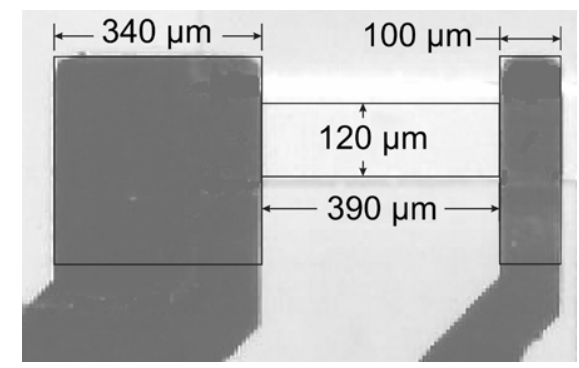

Figure 5. Dimensions of the electrodes. The left electrode is the signal electrode with a width of $390 \mu \mathrm{m}$. The right electrode (width $100 \mu \mathrm{m}$ ) was at ground potential. The inter-electrode gap is $390 \mu \mathrm{m}$. The pump channel (width $120 \mu \mathrm{m}$ ) spans the image horizontally.

thermal conductance of evolving small gas bubbles thermally insulating the vicinity of the spot.

The signal electrodes had the same geometry for both the directly and the indirectly heated structures (figure 5). A separate ground electrode was used for the indirectly heated structure. To ensure similar field conditions, the distance of the signal and the ground electrodes (heating element in case of the directly heated structure) was the same in both structures $(390 \mu \mathrm{m})$.

\subsection{Pump media}

The pump velocity was registered at conductivities of $0.1 \mathrm{~S} \mathrm{~m}^{-1}, 0.5 \mathrm{~S} \mathrm{~m}^{-1}, 0.9 \mathrm{~S} \mathrm{~m}^{-1}$ and $1.3 \mathrm{~S} \mathrm{~m}^{-1}$. The velocity was microscopically measured with a stop watch and grids on a video-monitor with scales from 25 to $100 \mu \mathrm{m}$. For flow measurements, latex particles ( $800 \mathrm{~nm}$ in diameter) were used as flow markers. These particles could be microscopically observed as small dark spots. Due to their small size they did not affect the flow and their sedimentation was very slow, allowing for long measuring times.

\subsection{Frequency dependence of the pump velocity}

Measurements in the $300 \mathrm{kHz}-52 \mathrm{MHz}$ frequency range were carried out in order to analyse the velocity dependence on the field frequency. Field electrode and heating voltage were fixed to $20 V_{\text {rms }}$ (corresponding to an effective field strength of $E=51282 \mathrm{~V} \mathrm{~m}^{-1}$ ) and $2.1 \mathrm{~V}$ to keep the pump field strengths and temperature gradient constant.
2.4. Field strength and heating voltage dependence of the pump velocity

To check the effect of electrode voltage on the pump velocity, the voltage between the field electrodes was varied from 0 to $35 V_{\text {rms }}$. The heating voltage was kept at $2.1 \mathrm{~V}$ to induce a constant temperature gradient. It was not possible to apply fields above $E=64103 \mathrm{~V} \mathrm{~m}^{-1}$ (corresponding to $25 V_{\text {rms }}$ ) for conductivities around $1.3 \mathrm{~S} \mathrm{~m}^{-1}$, since the induced temperature increase caused damage to the photoresist structures and the cover material.

To determine the influence of the temperature gradient, the heating voltage was varied from 1.1 to $3.6 \mathrm{~V}$ in steps of $0.5 \mathrm{~V}$ at a constant field-electrode voltage of $20 V_{\text {rms }}$.

\subsection{Finite element model}

A three-dimensional pump model was developed to model the observed effects (finite element program COMSOL Multiphysics; www.comsol.com). Aqueous properties were assumed for the pump medium, i.e. the temperature dependences of the electrical and thermal conductivities, the permittivity, the heat capacity and the density were assumed to be those of water. The respective parameters of the structural materials, i.e. heat capacities and thermal conductivities of glass, PDMS, photoresisist and platinum, were taken from the manufacturers' data sheets.

\section{Results}

\subsection{Thermal convections}

No thermal convections could be observed above the heating element when the ac-pump field was switched off. Only after switching on the heating element, a medium movement appearing as a small volume expansion was observed. The movement stopped within seconds. The finite element model predicted very little thermal convection (slower than $0.1 \mu \mathrm{m} \mathrm{s}^{-1}$ ) that could not be microscopically observed above the non transparent heating element. These observations prove that pumping is not driven by induced convections in our system.

\subsection{Frequency dependence of the pump velocity}

Results for the frequency dependence of the pump velocity are given in figure 6 . The pump velocities increase with frequency, exhibiting a peak around $20 \mathrm{MHz}$. No velocity plateaus were observed. Negligible changes of the peak frequencies were observed for conductivities above $0.5 \mathrm{~S} \mathrm{~m}^{-1}$. To summarize, the maximum pump velocities of the directly heated structures were nearly twice as high as those of the indirectly heated structures.

\subsection{Heating voltage dependence of the pump velocity}

Velocity data given in figure 7 show a nearly linear dependence of the two parameters. At heating voltages of $3.6 \mathrm{~V}$ the velocities for the indirectly and directly heated pumps were approximately $35 \mu \mathrm{m} \mathrm{s}^{-1}$ and $70 \mu \mathrm{m} \mathrm{s}^{-1}$, respectively. 

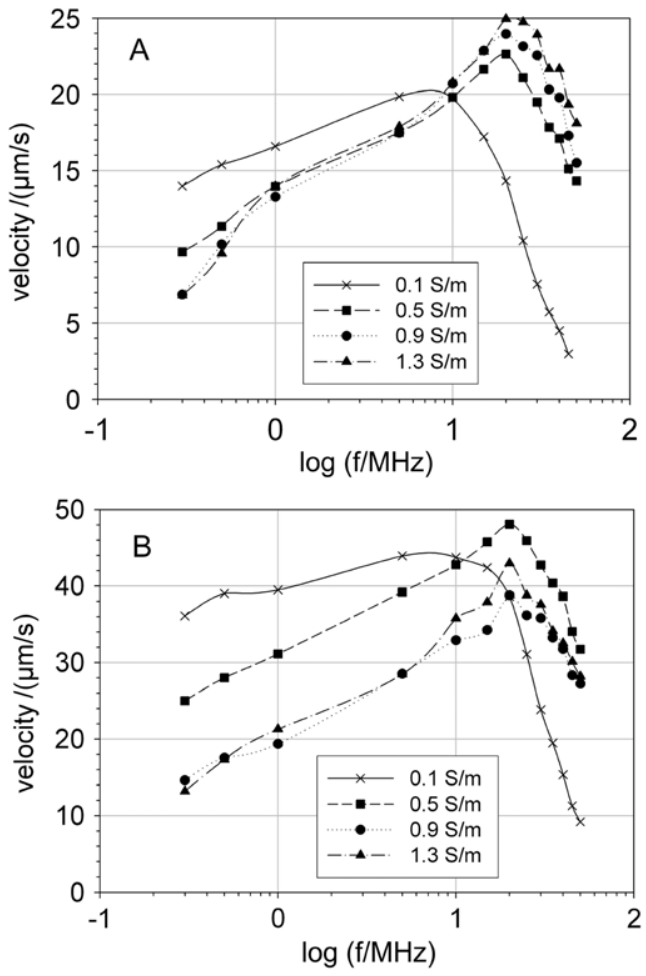

Figure 6. Plots of the pump velocities compared with the field frequencies for different medium conductivities $((a)$-indirectly, (b) - directly heated pump).
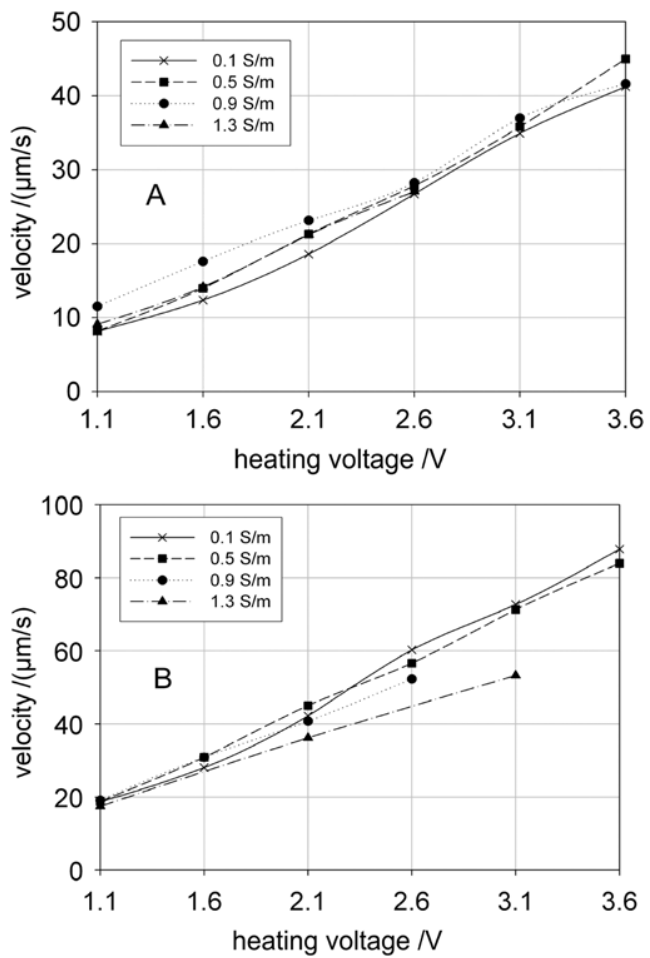

Figure 7. Plots of the pump velocities compared with the heating voltages for the indirectly $(a)$ and directly $(b)$ heated pumps. A nearly linear increase in the pump velocity with the heating voltage was observed for all media conductivities.
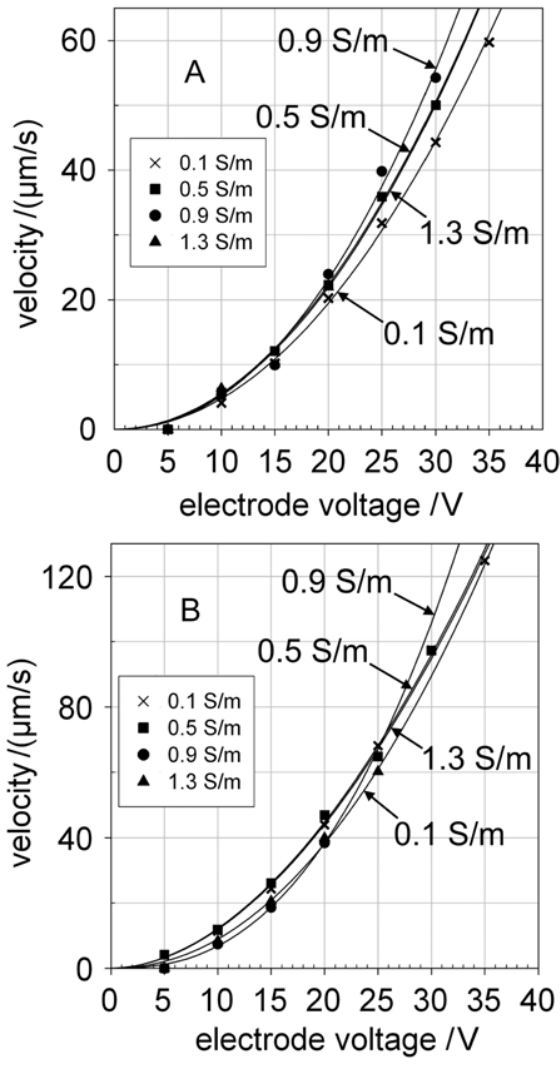

Figure 8. Plot of the pump velocity compared with the electrode voltage for different medium conductivities $((a)$-indirectly, (b) - directly heated pump). The graphs show a nearly quadratic dependence.

Table 1. Fitted parameters of equation (1) for the indirectly heated pump (figure 8(a)).

\begin{tabular}{lll}
\hline$\sigma\left(\mathrm{S} \mathrm{m}^{-1}\right)$ & Function & $R^{2}$ \\
\hline 0.100 & $v=0.0436 \cdot\left(V_{\text {rms }}\right)^{2.0373}$ & 0.9996 \\
0.503 & $v=0.0534 \cdot\left(V_{\text {rms }}\right)^{2.0151}$ & 0.9993 \\
0.900 & $v=0.0360 \cdot\left(V_{\text {rms }}\right)^{2.1591}$ & 0.9970 \\
1.304 & $v=0.0490 \cdot\left(V_{\text {rms }}\right)^{2.0379}$ & 0.9958 \\
\hline
\end{tabular}

\subsection{Field strength dependence of the pump velocity}

The results in figure 8 show a nearly quadratic increase in pump velocity with increasing electric field strength between the pump electrodes. This dependence persists for all medium conductivities. The curves for different conductivities are very similar within a pump structure.

To determine the exponent for the electrode voltages dependence, a theoretical function was fitted to the measuring points using SigmaPlot 9.0 (www.systat.com).

$$
v=a \cdot V_{\mathrm{rms}}^{b}
$$

Tables 1 and 2 present the fitted parameters of equation (1) for the indirectly and directly heated pumps at different medium conductivities. The means of exponent $b$ were 2.08 and 2.09, respectively. 
Table 2. Fitted parameters of equation (1) for the directly heated pump (figure $8(a)$ ).

\begin{tabular}{lll}
\hline$\sigma\left(\mathrm{S} \mathrm{m}^{-1}\right)$ & Function & $R^{2}$ \\
\hline 0.100 & $v=0.1547 \cdot\left(V_{\mathrm{rms}}\right)^{1.8872}$ & 0.9993 \\
0.503 & $v=0.1602 \cdot\left(V_{\mathrm{rms}}\right)^{1.8803}$ & 0.9989 \\
0.900 & $v=0.0218 \cdot\left(V_{\mathrm{rms}}\right)^{2.4944}$ & 0.9990 \\
1.304 & $v=0.0713 \cdot\left(V_{\mathrm{rms}}\right)^{2.0976}$ & 0.9964 \\
\hline
\end{tabular}

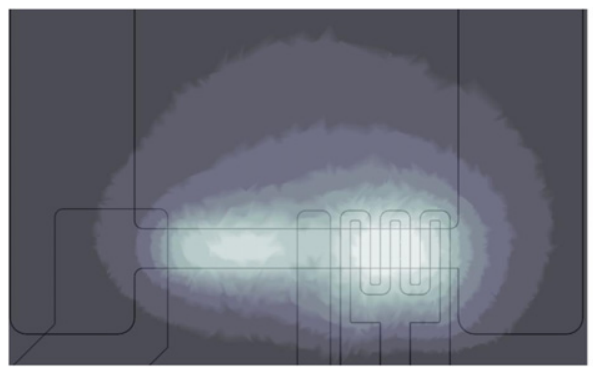

306

$300 \simeq$

294

Figure 9. Calculated temperature field in the indirectly heated pump calculated by COMSOL Multiphysics 3.2 at an external temperature $T_{0}=20^{\circ} \mathrm{C}$. Heating voltage, electrode voltage and medium conductivity were $2.1 \mathrm{~V}, 20 \mathrm{~V}_{\mathrm{rms}}$, and $0.9 \mathrm{~S} \mathrm{~m}^{-1}$, respectively.

\section{Discussion}

The increase observed in the pump velocities with the driving field frequency (figure 5) is similar to the results of Green et al (2000, 2002), Ramos et al (1998, 2003), Gonzales et al (2000), Ajdari (2000) and Wong et al (2004). Nevertheless, our experiments suggest negligible influence by ac electroosmosis in our pump structures. No pumping is observed for a separate application of either the heating or the ac-pump voltages. An instantaneous start of the pump flow could be observed when both voltages were applied, either after a consecutive or synchronous switch-on of the heating and the ac pump voltages. COMSOL modelling suggested a time constant in the ms-range for the onset of the pump streaming. This is in accordance with our experimental observations.

Higher temperature gradients induced by the heating element generate an increased force on the medium resulting in a higher pump velocity. On the other hand, a temperature increase by resistive Joule-heating of the pump medium induced by the pump field disturbs the build-up of the temperature gradient. The resulting superposition of two temperature fields may even reduce the temperature gradient and the resulting pump force especially for higher medium conductivities.

Figure 9 shows an example for the temperature field obtained for the indirectly heated pump structure. Two separate temperature hot spots, induced by Joule-heating of the medium and the heating element can be seen. A lower temperature is observed above the cooling ground electrode.

Figure 10 shows the temperature field for the directly heated pump structure. Please note that the two superimposed temperature fields are joined.

Temperature changes alter the physical medium characteristics in the channel. When the permittivity decreases the conductivity increases with temperature by about $2.3 \%$ per degree (Lide 2005, Mietchen et al 2002):

$$
\sigma=\sigma_{\text {fluid }}\left(1+\alpha_{\mathrm{T}}\left(T-T_{0}\right)\right) .
$$

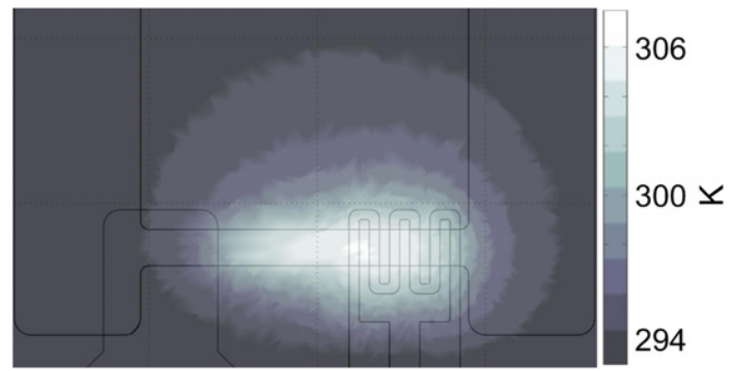

Figure 10. Calculated temperature field in the directly heated pump calculated by COMSOL Multiphysics 3.2 at an external temperature $T_{0}=20^{\circ} \mathrm{C}$. Heating voltage, electrode voltage and medium conductivity were $2.1 \mathrm{~V}, 20 \mathrm{~V}_{\text {rms }}$ and $0.9 \mathrm{~S} \mathrm{~m}^{-1}$, respectively.

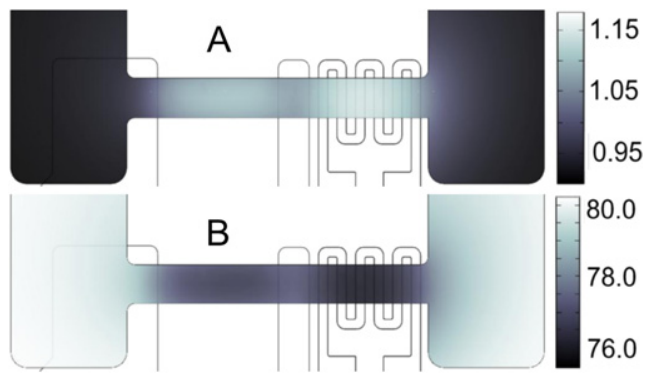

Figure 11. Electrical conductivity in $\mathrm{S} \mathrm{m}^{-1}(a)$ and relative permittivity $(b)$ of the medium in areas with different temperatures for the indirectly heated pump structure.

The permittivity decreases by approximately $0.4 \%$ per degree (Mietchen et al 2002; Ramos et al 1998). For our COMSOL calculations we used an improved analytical expression (equation (3), $R^{2}>0.999$ ):

$\varepsilon_{r}=7.3065 \times 10^{-4} \times T^{2}-0,795$

$$
\times T+250.613
$$

It was obtained by fitting standard permittivity values of water from a handbook in the temperature range from 273.15 to 373.15 K (Lide 2005).

The COMSOL calculations take feedback effects into account, such as the conductivity increase with temperature, leading to a higher absorption and in turn to a further temperature increase. As an example, figure 11 presents the permittivity (a) and conductivity (b) distributions obtained for the indirectly heated pump resulting from the equilibrium temperature field given in figure 9. Equations (2) and (3) were used to calculate the images.

The curves in figure 12 show the calculated temperature and field along the central axis of the pump channel. Please note that the irregularities in the curves of the electric field depend on the mesh quality of COMSOL Multiphysics 3.2, especially at the edge of the electrodes. An optimal mesh width was chosen (about 70000 knots for the model in figure 1) to reduce the computational time and to adapt the problem to the limited resources of the computer $(2.2 \mathrm{GHz}$ single core CPU, 4 GByte RAM).

The temperature gradient in the area of the ac-pump field is clearly higher in the directly heated pump than in the indirectly heated one. From equation (4), forces were calculated for a frequency of $1 \mathrm{MHz}$ (Green et al 2000, 2002, Ramos et al 1998, Gonzales et al 2000). This frequency is below the 

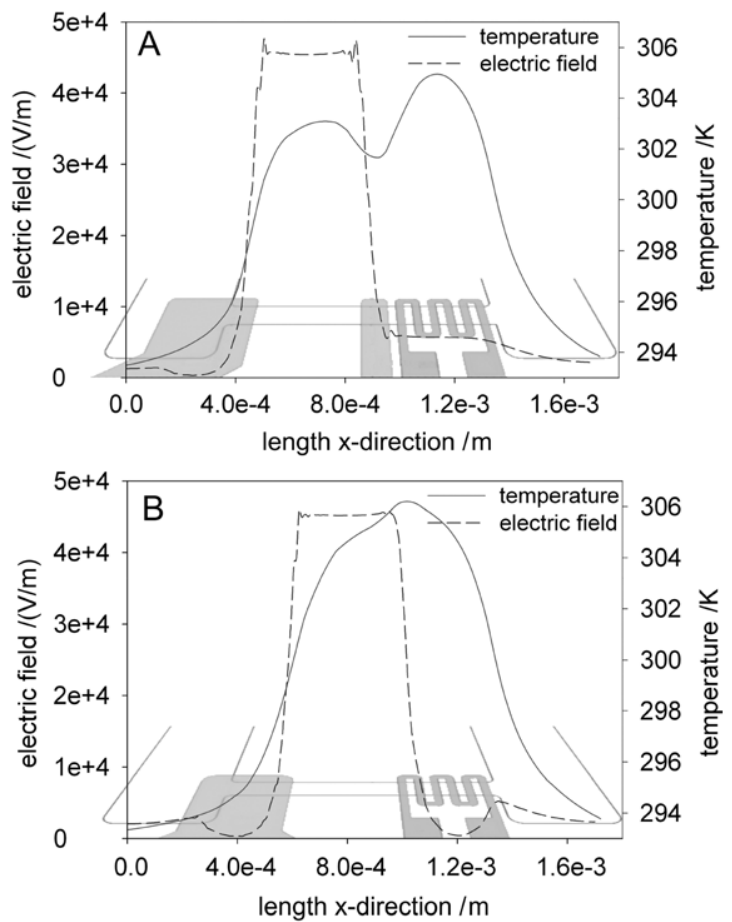

Figure 12. Overlay of geometry and the plots of temperature and electric field $((a)$-indirectly heated pump, $(b)$ - directly heated pump). Heating voltage $2.1 \mathrm{~V}$, field electrode voltage $20 V_{\mathrm{rms}}$ and conductivity $0.9 \mathrm{~S} \mathrm{~m}^{-1}$ at $T_{0}=20^{\circ} \mathrm{C}$.

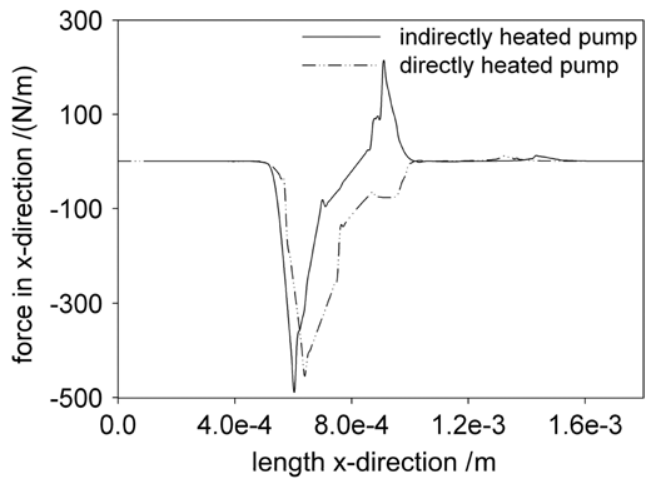

Figure 13. Resulting forces along the central axis of the pump channel in $\mathrm{x}$-direction obtained from equation (4) for indirectly and directly heated pumps (heating voltage $2.1 \mathrm{~V}$, field electrode voltage $20 V_{\text {rms }}$, medium conductivity $0.9 \mathrm{~S} \mathrm{~m}^{-1}, T_{0}=20^{\circ} \mathrm{C}$ ).

dispersion at our medium conductivities, i.e. the same forces were theoretically obtained for all frequencies below $1 \mathrm{MHz}$.

$F_{\mathrm{e}}=-\frac{1}{2}\left[\left(\frac{\nabla \sigma}{\sigma}-\frac{\nabla \varepsilon}{\varepsilon}\right) \cdot \vec{E}_{0} \frac{\varepsilon \vec{E}_{0}}{1+(\omega \tau)^{2}}+\frac{1}{2}\left|E_{0}\right|^{2} \nabla \varepsilon\right]$.

The resulting forces of both pump types are shown in figure 13. A comparison of figures $12(a)$ and $(b)$ and 13 shows a clear connection between temperature distribution and forces in the areas of the ac-pump field.

The smoother temperature gradient of the directly heated pump in the regions of the ac-pump field results in an increased pump effect (compared with figure 12). Areas of an inverse gradient generate counter-acting forces especially in the indirectly heated pump leading to a lower velocity (figure 13). Positive forces of about $100 \mathrm{~N} \mathrm{~m}^{-3}$ and negative forces of about $200 \mathrm{~N} \mathrm{~m}^{-3}$ were calculated for the indirectly heated pump. In the directly heated pump only negative forces of about $200 \mathrm{~N} \mathrm{~m}^{-3}$ were obtained.

These numerical results are in accordance with the experiments. Measured pump velocities differed by a factor of about 2 for both types of pumps (figure 6). Nevertheless, the absolute velocities calculated for both pumps are lower than the experimental values. An error-factor of about 12.5 was obtained for all medium conductivities and all pump structures used. We tested that the error-factor is sensitive to the mesh width. Because of the limited computer power a thorough investigation of this effect is not yet possible. Another possible source of error is the experimental determination of the temperature distribution. A direct comparison of theoretical and experimental results would require temperature probes much smaller than the channel width $(100 \mu \mathrm{m}$ at a height of $60 \mu \mathrm{m})$. With radii from 100 to $150 \mu \mathrm{m}$ available temperature probes are relatively large. Smaller temperature probe particles (Jaeger 2007) were not commercially available at the time of our experiments.

We suppose that a major contribution to the error-factor arises from the coarse description of the pump effect by equation (4). A hint to possible missing terms in the equation comes from the correct reflection of the frequency dependence with two velocity plateaus at low conductivities $\left(<0.1 \mathrm{~S} \mathrm{~m}^{-1}\right)$. This was also found for other pump structures (paper in preparation). Nevertheless, in these structures the dispersion frequency for the transition between the two plateaus was conductivity-dependent as described by equation (4). By contrast, a nearly linear increase in the pump velocity over the logarithm of frequency was observed in the present pumps instead of the predicted plateau at low frequencies (figure 6). Further, the dispersion frequency was roughly constant above a conductivity of $0.1 \mathrm{~S} \mathrm{~m}^{-1}$.

Equation (4) predicts a velocity increase with the square of the pump field strength. This relation is largely correct. In both pumps the exponent is very close to 2 (2.09 and 2.08, respectively for the directly and indirectly heated pumps). A value of 2.44 was described for travelling wave pumps (Gimsa et al 1997). These deviations suggest that an unknown process is involved in the pump effect. We assume that equation (4) needs further consideration.

In summary, our new micro-pump designs generate reproducible pump effects. Pumping of the medium is performed without any mechanical moving parts. The pumps combine high flow rates in a pump channel of constant width with an extremely simple construction. Their low fabrication cost, low power consumption and efficient use of chemicals make them interesting for applications in micro-factories on chip and $\mu$ TAS systems in chemical, biological or medical micro-fluidic systems.

\section{Acknowledgments}

The authors would like to thank Robert Sleigh for his help with the language. 


\section{References}

Ajdari A 2000 Pumping liquids using asymmetric electrode arrays Phys. Rev. E 61 R45-8

Bourouina T, Bosseboeuf A and Grandchamp J-P 1997 Design and simulation of an electrostatic micropump for drug-delivery applications J. Micromech. Microeng. 7 186-8

Ehrlich R M and Melcher J R 1982 Bipolar model for traveling-wave induced nonequilibrium double-layer streaming in insulation liquids Phys. Fluids 25 1785-93

Fuhr G, Glaser R, Hagedorn R, Gimsa J, Benecke W and Wagner B 1990 Dielektrisches Mikromechanisches Element DE 4003 114 A1

Fuhr G, Hagedorn R, Müller T, Benecke W, Wagner B and Gimsa J 1991 Asynchronous traveling-wave induced linear motion of living cells Studia Biophys. 140 79-102

Fuhr G, Hagedorn R, Müller T, Benecke W and Wagner B 1992 Microfabricated electrohydrodynamic (EHD) pumps for liquids of higher conductivity J. Microelectromech. Systems 1 141-6

Fuhr G, Schnelle T and Wagner B 1994 Travelling wave-driven microfabricated electrohydrodynamic pumps for liquids J. Micromech. Microeng. 4 217-26

Gimsa J and Holtappels M 2003 Pumpe mit mindestens einer Pumpkammer und Elektroden zum Erzeugen eines elektrischen Wechselfeldes DE 10329 979.3-15; DE 200400 1380; PCT 04 738 826.9-2315

Gimsa J, Eppmann P and Prüger B 1997 Introducing phase analysi light scattering for dielectric characterization: measurement of traveling-wave pumping Biophys. J. 73 3309-16

Gonzales A, Green N G, Ramos A, Castellanos A and Morgan H 2000 Fluid flow induced by nonuniform ac electric fields in electrolytes on microelectrodes: II. A linear double-layer analysis Phys. Rev. E 61 4019-28

Green N G, Ramos A, Gonzales A, Morgan H and Castellanos A 2000 Fluid flow induced by nonuniform ac electric fields in electrolytes on microelectrodes: I. Experimental measurements Phys. Rev. E 61 4011-18

Green N G, Ramos A, Gonzales A, Morgan H and Castellanos A 2002 Fluid flow induced by nonuniform ac electric fields in electrolytes on microelectrodes: III. Observation of streamlines and numerical simulation Phys. Rev. E 66026305

Hagedorn R, Fuhr G, Müller T and Gimsa J 1992 Travelling-wave dielectro-phoresis of microparticles Electrophoresis 13 49-54

Lide D R 2005 Handbook of Chemistry and Physics (Boca Raton, FL: CRC Press)

Jaeger M S, Mueller T and Schnelle T 2007 Thermometry in dielectrophoresis chips for contact-free cell handling J. Phys. D: Appl. Phys. 40 95-105

Melcher J R 1966 Traveling-wave induced electroconvection Phys. Fluids 9 1548-55

Melcher J R and Firebaugh U S 1967 Traveling wave bulk electroconvection induced across a temperature gradient $P$ hys. Fluids 10 1178-85

Melcher J R and Taylor G I 1969 Electrohydrodynamics: a review of the role of interfacial shear stresses Annu. Rev. Fluid Mech 1 111-46

Mietchen D, Schnelle T, Müller T, Hagedorn R and Fuhr G 2002 Automated dielectric single cell spectroscopy-temperature dependence of electrorotation J. Phys. D: Appl. Phys. 35 1258-70

Müller T, Arnold W M, Schnelle T, Hagedorn R, Fuhr G and Zimmermann U 1993 A traveling-wave micropump for aqueous solutions. Comparison of $1 \mathrm{~g}$ and $\mu \mathrm{g}$ results Electrophoresis 14 764-72

Ramos A, Morgan H, Green N G and Castellanos A 1998 Ac electrocinetics: a review of forces in microelectrode structures J. Phys. D: Appl. Phys. 31 2338-53

Ramos A, Gonzalez A, Castellanos A, Green N G and Morgan H 2003 Pumping of liquids with ac voltages applied to asymmetric pairs of microelectrodes Phys. Rev. E 67056302

Reyes D R, Iossifidis D, Auroux P-A and Manz A 2002 Micro Total Analysis Systems: I. Introduction, Theory, and Technology Anal. Chem. 74 2623-36

Wang X-B, Huang Y, Becker F F and Gascoyne P R C 1994 A unified theory of dielectrophoresis and travelling wave dielectrophoresis J. Phys. D: Appl. Phys. 27 1571-4

Wong P K, Wang T-H, Deval J H and Ho C-M 2004 Electrokinetics in micro devices for biotechnology applications IEEE/ASME Trans. Mechatronics. 9 1083-4435 